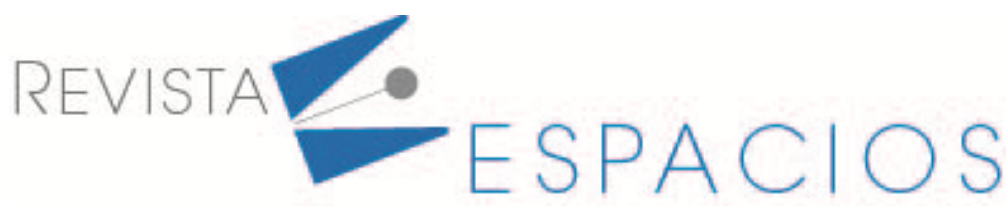

\title{
Contraste de resultados para validación de una escala Likert usando un enfoque tradicional y nuevas recomendaciones
}

\author{
Likert scale validation results using tradicional approach and new recommendations \\ contrast
}

CONTRERAS, Carolina N. ${ }^{1}$

GONZÁLEZ, Ricardo I. ${ }^{2}$

\begin{abstract}
Resumen
En este artículo se contrasta los resultados de un análisis exploratorio siguiendo recomendaciones actualizadas (i.e., Análisis Factorial Exploratorio, AFE), y los criterios clásicos (i.e., Análisis de Componentes Principales ACP). De igual forma se contrasta el tipo de rotación (i.e., Oblicua u Ortogonal). Se evidencian las diferencias en los resultados y la importancia de considerar las recomendaciones recientes. En especial, las características de la muestra determinan la selección de los procedimientos.
\end{abstract}

Palabras clave: ACP, AFE, rotación, número de factores.

\begin{abstract}
This article contrasts the results of an exploratory analysis following most recent recommendations (i.e., Exploratory Factor Analysis, EFA), and the classical criteria (i.e., Principal Component Analysis, PCA). The type of rotation (i.e., Oblique or Orthogonal) is also contrasted.

The differences in the results and the importance of considering recent recommendations are evidenced. In particular, the characteristics of the sample determine the selection of the procedures.

Key words: PCA, EFA, rotation, number of factors
\end{abstract}

\section{Introducción}

Frente a la metodología para la validación de escalas psicométricas existen técnicas más usadas que otras, ya sea, por su facilidad de implementación, por su tradición, divulgación y acceso en determinadas disciplinas, entre otros. Sin embargo, dicha popularidad ha sido puesta en discusión en algunas publicaciones desde los aspectos metodológicos (Lloret-Segura, et al., 2014) que involucran el tipo de análisis estadístico (Ferrando, Ledesma, \& Tosi, 2019) y el tipo de software a utilizar (Lloret-Segura, et al., 2017). Frente al análisis de una escala tipo Likert el investigador se ve enfrentado a tomar la decisión de utilizar las técnicas más clásicas, ampliamente usadas y

\footnotetext{
${ }^{1}$ Estudiante Magister. Facultad de Educación. Universidad Católica de la Santísima Concepción. Chile. Email: ccontreras@magisteredu.ucsc.cl 2 Docente. Departamento de Currículum y Evaluación, Facultad de Educación. Universidad Católica de la Santísima Concepción. Chile. Email: rgonzalez@ucsc.cl
} 
divulgadas (i.e., Análisis de Componentes Principales ACP), o usar las recomendaciones que desaconsejan estas técnicas.

\subsection{Objetivo}

El objetivo de este artículo es contrastar los resultados de un análisis considerado clásico y uno que siga las recomendaciones más recientes a modo de ejemplo para investigadores nóveles. Como objetivos específicos se contrastó el Análisis de Componentes Principales (ACP) con Análisis Factorial Exploratorio (AFE). Además, se contrastó ACP y AFE donde se usaron diferentes tipos de rotación oblicuo u ortogonal.

Para seleccionar las recomendaciones de la literatura reciente se usó el criterio de seleccionar el tipo de análisis según las características específicas de la escala.

Primeramente, veremos la comparación de resultados entre el ACP y AFE. Dicha discusión podría presuponer que ambos métodos son lo mismo, por lo tanto, entregarán el mismo resultado. No obstante, no es así. En la tradición se ha planteado y usado el ACP como un método de extracción de factores lo que ha generado esta confusión (Ferrando, Ledesma, \& Tosi, 2019). No obstante, hoy existen publicaciones que son enfáticas en señalar que el ACP no es un método que permita descubrir la variable latente, sino que permite la reducción de componentes (Lloret-Segura et al., 2014).

Segundo, presentaremos las diferencias de ACP y AFE según el tipo de rotación. Dicho punto, nos contrapone entre la recomendación de un método oblicuo u otro ortogonal. Se realiza la comparación por la rotación específica Varimax y Oblimin. En las recomendaciones de la literatura actual (Lloret-Segura et al., 2014) se debe analizar exhaustivamente las características de los datos para determinar el tipo de rotación. En este caso dichas características corresponden a la psicometría principalmente.

\section{Metodología}

Los datos utilizados corresponden a una investigación para la validación de una escala tipo Likert.

La escala cuenta con 53 ítems (Anexo 1) que nacen de la teoría de autorregulación de los aprendizajes de Zimmerman (Panadero \& Alonso, 2014). Se mide frecuencia con 7 opciones que va desde "nunca" a "siempre". Se divide en tres la escala con tres consignas que anticipan los momentos de la tarea: Antes de empezar la investigación en Lenguaje y Comunicación... ; Mientras realizo la investigación en Lenguaje y Comunicación...; y , Una vez terminada la investigación en Lenguaje y Comunicación. Para realizar el contraste se utilizó una muestra de 279 personas.

Se realizaron las pruebas de normalidad, las pruebas de fiabilidad para consistencia interna y las pruebas de Kaiser-Meyer-Olkin y la prueba de esfericidad Bartlett's. Una vez terminado esto se realizaron las pruebas estadísticas ACP y AFE.

\subsection{Elección de la Metodología de análisis preliminares}

La muestra tiene características que son particulares como son no tener normalidad y tener un sesgo negativo. Dichas características posicionan y plantan al investigador frente a una cuestión recurrente en la validación, búsqueda y toma de decisiones para al análisis de los datos. En este caso, seguimos las indicaciones de la literatura cuando no se obtiene el resultado de los supuestos de normalidad (Freiberg, et al., 2013) que indican que la violación de este supuesto en esta muestra no trae consecuencias estadísticamente significativas en los resultados. 
La confiabilidad o consistencia interna fue medida comparando alpha de Cronbach, McDonald's omega (Ventura \& Caycho, 2017) y alfa ordinal (Domínguez, 2012). En este trabajo no se profundiza en estas diferencias, solo se dirá que todas ellas fueron favorables. No obstante, siguiendo las recomendaciones de la literatura, para AFE, se informa el alfa ordinal que se sugiere, para las escalas policóricas (véase tabla 3, fila AFE), como el más indicado de .9 (Freiberg, et al., 2013)

La prueba de Kaiser-Meyer-Olkin es muy buena (.957) ya que es más cercana a 1 y la prueba de esfericidad Bartlett's statistic fue $10172,451(\mathrm{gl}=1368$; Sig $=.0001)$. Ahora bien, las siguientes decisiones a tomar corresponden a las características de la muestras. Pero, también a los objetivos específicos que se espera lograr.

\subsection{Metodología para la estimación del número de factores}

El problema del camino a seguir para un análisis factorial es compleja para el investigador novel cuando ante él se presentan diferentes escuelas, tradiciones, publicaciones, software, entre otros. En este caso, se tomaron dos modelos para comparar: La tradición y popularidad de manuales encontrados para análisis factoriales, y, por otro lado, se considera la literatura científica que cuestiona dicha tradición.

La publicación "El análisis factorial exploratorio de los ítems: una guía práctica, revisada y actualizada" (LloretSegura, et al., 2014) es muy detallada en las recomendaciones y es explícita en desaconsejar algunas prácticas tradicionales. Puntualiza, además, cuál es el correcto uso de cada uno de estos análisis según el objetivo que se quiere lograr.

Es de interés de esta investigación contrastar los resultados para corroborar la literatura. La nueva recomendación que consideraremos primordial será, tener presente las características de la escala para la selección de los métodos de análisis.

Las primeras características que se consideraron fueron que la escala usada es policórica y utiliza constructos de la psicología. Frente a esta característica la literatura indica que es posible encontrar dificultades al ser escalas menos precisas y/o estables (Ferrando, et al., 2019). Justamente, la escala usada no cumple con los supuestos de normalidad. Frente a esta característica la literatura refiere que la violación del supuesto de normalidad es una característica a considerar y estudiar, pero no es un impedimento para realizar los análisis, ya que si cumple con requisitos como el número de muestras (entre otros) no alterará los resultados (Freiberg, et al., 2013).

Con respecto a la consistencia interna la tradición usa el índice de alfa de Cronbach; mientras que, para las escalas que son policóricas, las nuevas recomendaciones son usar el alfa ordinal (Freiberg, et al., 2013). Por otra parte, existen otros textos que recomiendan omega (Hayes \& Coutts, 2020). En este caso, como el objetivo es contrastar resultados en el ACP oblicuo usaremos omega y en el AFE oblicuo usaremos alfa ordinal (Domínguez, 2014).

En lo que respecta a las correlaciones para el AFE la tradición usará medidas de Pearson (Freiberg, et al., 2013). Mientras que las nuevas recomendaciones la desaconsejan para escalas como la de este estudio (policóricas o tetracóricas) (Freiberg, et al., 2013). Frente a esto, como solución se presenta un método robusto, en este caso, Robust Unweighted Least Squares, según el número de la muestra (Ferrando, et al., 2019).

Sobre la tradición y el tipo de rotación, se puede decir que Varimax, por mucho tiempo ha sido la opción de los expertos (Freiberg, et al., 2013). No obstante, en la literatura reciente se presenta que para estudios exploratorios y constructos psicológicos es preferible los métodos ortogonales (Ferrando, et al., 2019).

El resultado del ACP, tradicionalmente es usado como método de extracción de factores, pero no es tal (LloretSegura et al., 2014). Este debe ser usado para análisis de congruencia de las variables con el constructo y para reducción de variables. La explicación de esto corresponde al tipo de varianza usado en el análisis: la varianza 
total (Ferrando \& Anguiano, 2010). Por otra parte, el AFE utiliza la varianza común para hallar la variable latente, por lo cual, será más aconsejable por sobre el ACP (Ferrando \& Anguiano, 2010). Véase en la tabla 1 el contraste entre ACP y AFE con los respectivos tipos de varianzas que usan y los usos que deberían darse de acuerdo a la recomendaciones actualizadas de la literatura referida.

Tabla 1

Comparación de ACP y AFE

\begin{tabular}{|c|c|}
\hline ACP & AFE \\
\hline $\begin{array}{c}\text { Usa la varianza total (Lloret, Ferreres, Hernández, Tomás, } \\
\text { 2014) }\end{array}$ & $\begin{array}{c}\text { Usa la varianza común, Considerando la varianza del error } \\
\text { (Lloret, Ferreres, Hernández, Tomás, 2014) }\end{array}$ \\
\hline $\begin{array}{c}\text { Sirve para } \\
\text { Reducción de variables (Lloret et al., 2014) }\end{array}$ \\
$\begin{array}{c}\text { Ver la congruencia entre los componentes o compuestos } \\
\text { con el constructo (Lloret et al., 2014) }\end{array}$ & $\begin{array}{c}\text { Hallar variables latentes (Lloret et al., 2014) } \\
\text { Descubrir constructo que subyace a las variables } \\
\text { manifiestas. (Lloret et al., 2014) }\end{array}$ \\
\hline $\begin{array}{c}\text { No debe ser usada para: } \\
\text { Extracción de factores (Ferrando, Ledesma, \& Jeremías, } \\
\text { 2019) (Lloret et al., 2014) (Ferrando, et al., 2010) }\end{array}$ \\
\hline
\end{tabular}

Fuente: Tabla de elaboración propia

Otra recomendación corresponde a los software estadísticos (Ferrando, Ledesma, \& Tosi, 2019) . Hoy existen variedad de nuevos programas que se enfrentan a los tradicionales, como SPSS, y su uso indiscriminado(Ferrando, Ledesma, \& Tosi, 2019). En este caso, FACTOR (Lorenzo \& Ferrando, 2017) es un programa que aparece en la literatura como una opción gratuita para escalas policóricas o tetracóricas (Freiberg, et al., 2013). Dicho programa es utilizado para el AFE obedeciendo a las recomendaciones de la literatura (LloretSegura et al., 2014)

\section{Resultados y discusión}

La comparación de los resultados de los ACP usando 2 tipos diferentes de rotaciones, oblicua y ortogonal evidenció el contraste entre la cantidad de los componentes, la reducción de variables, y, la congruencia teórica.

Por otra parte, el contraste de los resultados del ACP y AFE evidencia que ambos tienen objetivos de análisis diferentes.

Por lo tanto, frente a la problemática primaria, el investigador podrá seleccionar de acuerdo a su objetivo y las características de su escala y muestra.

\subsection{Resultados de número de componentes con ACP}

El resultado del ACP oblicuo, Oblimin evidenció una mayor reducción de los reactivos. De los 53 reactivos quedan 31 (Anexo 2). Por otra parte, tiene menos congruencia con el constructo de origen al eliminar el componente correspondiente a los reactivos de creencias y dejar 4 componentes. Véase en la tabla 2 la columna de rotación Oblimin.

Mientras, el ACP realizado con rotación ortogonal Varimax nos da mayor congruencia al dar 6 componentes. Aunque uno de ellos está unificando autorreflexión y otro está dividiendo planificación. Por otra parte, frente a la reducción de reactivos, de los 53 reactivos quedan 41 . Véase en la tabla 2 la columna de rotación varimax. 
Tabla 2

Componentes resultantes de los ACP y la cantidad de reactivos según las rotaciones.

\begin{tabular}{|c|c|c|}
\hline Reactivos del constructo original & Rotación varimax & Rotación Oblimin \\
\hline \multirow[t]{2}{*}{15 Planificación, análisis de la tarea } & $\begin{array}{l}\text { Lo divide en dos componentes } \\
5 \text { planificación estratégica }\end{array}$ & \multirow[t]{2}{*}{7 de planificación estratégica } \\
\hline & 4 Análisis de mis conocimientos & \\
\hline 7 Planificación, creencias & 5 creencias & \\
\hline 11 Ejecución, auto observación & 11 auto observación & 9 auto observación \\
\hline 10 Ejecución, auto control & 7 auto control & 7 de autocontrol \\
\hline 7 Autorreflexión, Autojuicio & \multirow{2}{*}{$\begin{array}{l}\text { Unifica los dos grupos } \\
9 \text { Autorreflexión } \\
\text { (6 autojuicio-3 auto reacción) }\end{array}$} & \multirow{2}{*}{$\begin{array}{c}8 \text { Autorreflexión } \\
\text { (Autojuicio y autoreacción) }\end{array}$} \\
\hline $\begin{array}{l}3 \text { Autorreflexión, auto reacción } \\
\text { cognitiva y emocional }\end{array}$ & & \\
\hline
\end{tabular}

Por otra parte, dentro de la tradición también se usa la regla de Kaiser que determina que los factores serán los valores mayores a 1. El mismo criterio se usa para el gráfico de sedimentación o scree test, los cuales se desaconsejan en la literatura actualizada (Lloret-Segura et al., 2014). En esta investigación los gráficos nos muestran para la rotación Oblimin 4 valores sobre 1 (Véase en el gráfico 2). Por otro lado, el gráfico de sedimentación que usó rotación Varimax hay 6 valores sobre 1 (Véase en el gráfico 1).

\section{Gráfico 1}

Gráfico de sedimentación ortogonal

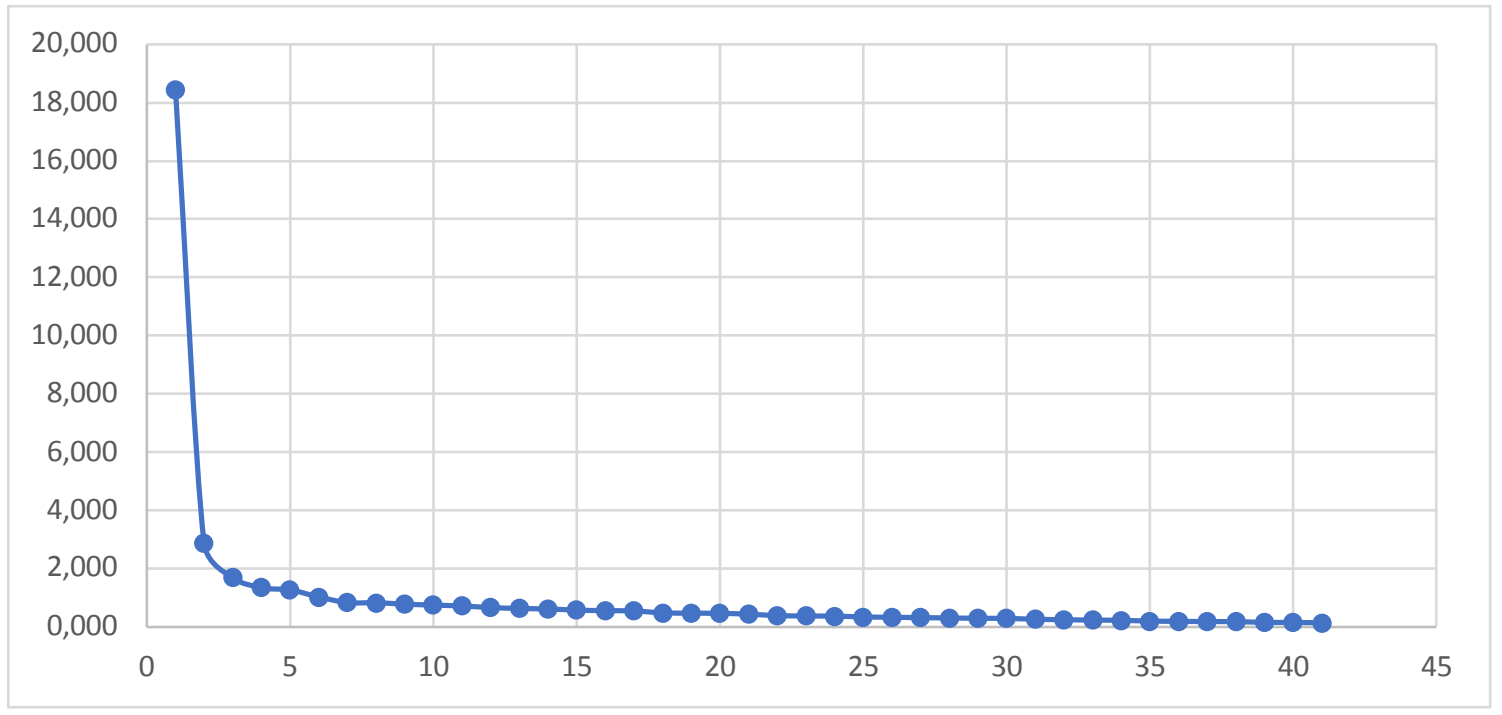

Autovalores mayores a 1 son 6 . La especificación de ellos es la siguiente:

$\begin{array}{ll}1 & 18,443 \\ 2 & 2,857 \\ 3 & 1,695 \\ 4 & 1,341 \\ 5 & 1,264 \\ 6 & 1,024\end{array}$


Gráfico 2

Gráfico de sedimentación oblicua

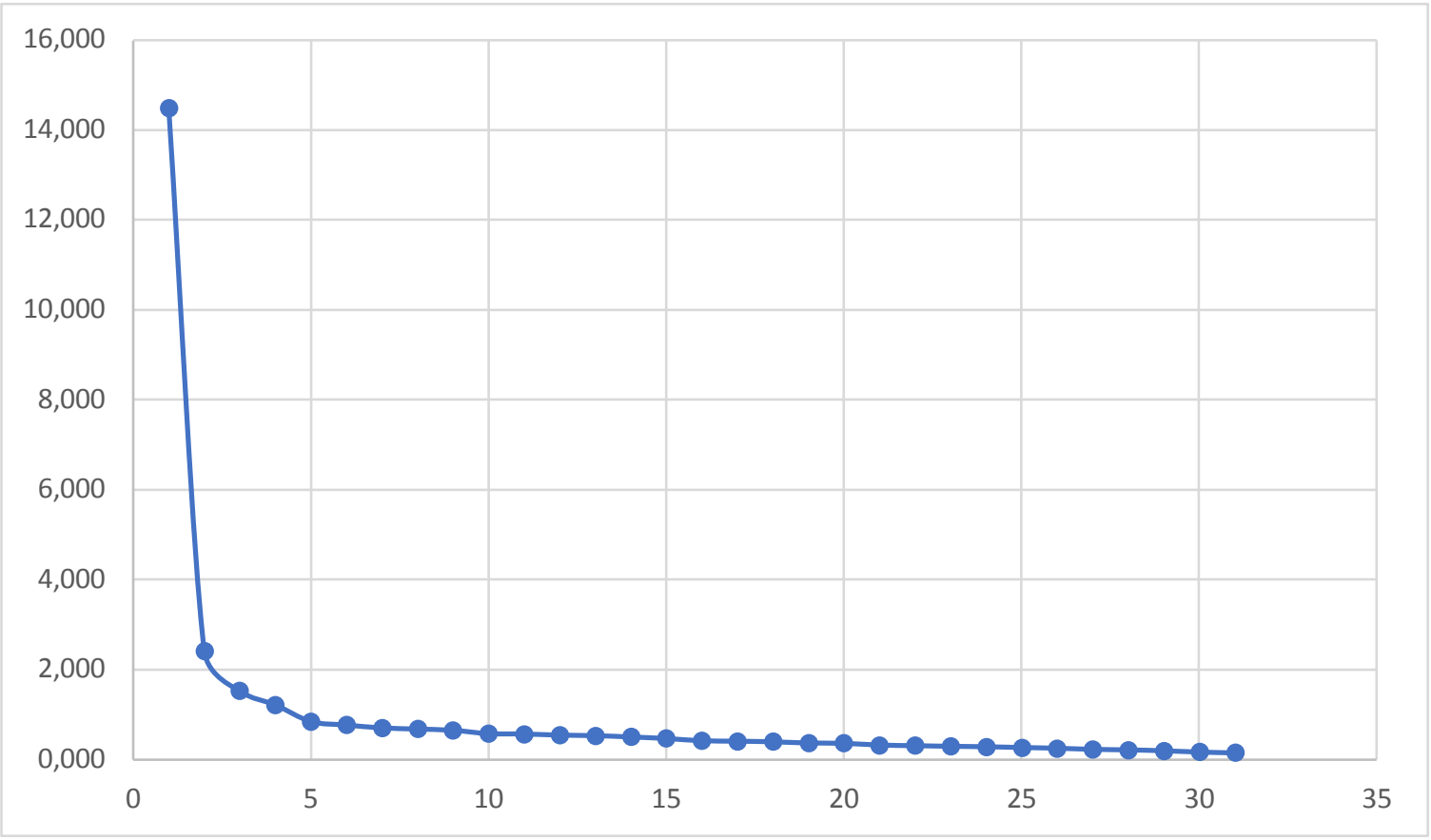

Autovalores mayores a 1 son 4, La especificación de estos es la siguiente:

$1 \quad 14,481$

$2 \quad 2,413$

31,526

$4 \quad 1,212$

\subsection{Resultado de congruencia teórica ACP}

\subsubsection{ACP ortogonal Varimax}

Primeramente, el ACP ortogonal Varimax (véase anexo 3) entrega un análisis preliminar que indica cierta congruencia teórica (véase tabla 2) entre el constructo (Zimmerman, 2010) y la escala (Véase anexo 1). Este, identificó dimensiones semejantes, según la varianza total, pero no idénticas a las usadas en la elaboración de la escala. Esto es, de las tres etapas del ciclo de autorregulación se determina 6 dimensiones para la elaboración de la escala y el ACP agrupó 6 dimensiones, pero dividió una de estas y unificó otra.

El ACP evidenció concordancia teórica con la primera fase "planificación". Se identifican dos de las dimensiones en "Análisis de la tarea": La teoría primigenia propone que en el "Análisis de la tarea", establecemos objetivos y niveles de perfección con los que haremos la tarea como también la planificación y visualización de las estrategias que se usarán para lograr realizarla. Es interesante ver como en el análisis fueron separados los reactivos: por una parte, los que se referían a lo que sabían sobre investigación para enfrentar la tareas, y por otra parte, los reactivos que se referían a planificar estrategias para realizar la investigación. En tanto, la dimensión "creencias", de esta misma fase "planificación", queda con congruencia teórica con 5 reactivos.

La fase del ciclo "ejecución de la tarea", en su dimensión de "auto observación" se agrupa claramente con 11 reactivos en el ACP. No sucede lo mismo con "autocontrol" donde son eliminados 3 de sus reactivos. 
La fase "autorreflexión" se construye en la escala realizando la distinción teórica entre "autojuicio" y "autorreacción". No obstante, no se profundiza en todas las subdimensiones de estas. Los resultados unificaron estos dos "autojuicio" y "auto reacción" en un solo componente.

\subsubsection{ACP oblicuo Oblimin}

Por otro lado, el ACP oblicuo (véase anexo 2), Oblimin, también evidenció de la primera fase planificación concordancia teórica semejante (véase tabla 2). Sin embargo, en el análisis fueron separados los reactivos de análisis de la tarea: por una parte, los que se referían a lo que sabían los alumnos sobre investigación para enfrentar las tareas, y por otra parte, los reactivos que se referían a planificar estrategias para realizar la investigación. Sin embargo, mientras se avanzó en el análisis de los reactivos sobre lo que sabían, fueron eliminados por ambigüedad o baja carga.

En tanto, la dimensión de "creencias", no se ordena en un componente. Aunque, logra su ordenación con rotación Varimax, no es tal con rotación Oblimin.

En la fase del ciclo de ejecución de la tarea, los componentes no presentan dificultades. Su dimensión de auto observación evidencia clara congruencia con 11 reactivos (solo pierde 2 reactivos) y con autocontrol donde son eliminados 3 de sus reactivos.

La fase de autorreflexión se comporta de igual forma que con la rotación Varimax y se unifica en un componente.

\subsection{Contraste resultado ACP y AFE}

En el desarrollo de este artículo ya se expuso que el ACP es un análisis que no permite descubrir factores, a pesar del uso que se ha dado para ello. En contraste, está el AFE como el análisis recomendado. En los resultados, se evidencia la diferencia de estos. Se puede apreciar un claro contraste entre los resultados (véase tabla 3), ya que el AFE permite encontrar dos factores (véase en la fila AFE de la tabla 3), mientras el ACP señala el doble o más componentes, dependiendo el tipo de rotación (véase en la fila ACP de la tabla 3).

Tabla 3

Comparación componentes

de ACP y factores de AFE

\begin{tabular}{|c|c|c|c|c|}
\hline \multirow[b]{2}{*}{ ACP } & \multicolumn{2}{|c|}{ Rotación Ortogonal Varimax } & \multicolumn{2}{|c|}{ Rotación Oblicua Oblimin } \\
\hline & $\begin{array}{l}\text { Escala con } 41 \text { reactivos } \\
6 \text { componentes }\end{array}$ & $\begin{array}{l}\text { Alfa de } \\
\text { cronbach }\end{array}$ & $\begin{array}{c}\text { Escala con } 31 \text { reactivos } \\
4 \text { componentes }\end{array}$ & $\begin{array}{c}\text { Omega } \\
\text { Mcdonald }\end{array}$ \\
\hline & Auto-observación & .939 & Auto-observación & .933 \\
\hline & Autorreflexión & .907 & Autorreflexión & .907 \\
\hline & Análisis de la t. 1 & .873 & Análisis de la tarea & .899 \\
\hline & Creencias & .849 & \multirow[t]{3}{*}{ Auto- Control } & \multirow[t]{3}{*}{.880} \\
\hline & Autocontrol & .882 & & \\
\hline & Análisis de la t. 2 & .845 & & \\
\hline \multirow[t]{3}{*}{ AFE } & $\begin{array}{c}\text { Escala con } 38 \text { reactivos } \\
2 \text { Factores }\end{array}$ & Alfa ordinal & $\begin{array}{c}\text { Escala con } 22 \text { reactivos } \\
2 \text { factores }\end{array}$ & Alfa ordinal \\
\hline & Dimensión Planificación y ejecuta & .972 & Dimensión Planificación y ejecuta & .946 \\
\hline & Dimensión autorreflexión & .921 & Dimensión autorreflexión & .929 \\
\hline
\end{tabular}

El AFE es un conjunto de métodos estadísticos multivariados de interdependencia con los cuales se identifican factores subyacentes (Pérez \& Medrano, 2014) que agrupan ítems interrelacionados. Es decir, que un conjunto de variables se encuentran relacionadas por medio de lo que conocemos como rasgo o factor latente. El 
constructo usado para la construcción de la escala, "autorregulación", nos da tres fases cíclicas: Planificación de la tarea, Ejecución de la tarea y Autorreflexión en los resultados (Zimmerman, Kitsantas, \& Campillo, 2005). En esta investigación se evidencia que el AFE unifica dos partes de las tres del ciclo de autorregulación (Zimmerman, et al., 2005) y deja como variable la tercera. Podemos concluir que, el ciclo de autorregulación, con tres partes, no se comporta como tal en la muestra y en la escala. Ya que, está separado en 2 factores y no en los 3. Específicamente, planificación y ejecución que en la tarea de investigación son vistos como una variable.

Cabe resaltar, además, un contraste sobre el constructo. Se evidencian subdimensiones de la teoría en el ACP, donde se pueden ver componentes correspondientes a subdimensiones de las tres fases (creencias, autocontrol, auto observación, entre otros). Pero, esto no es evidenciado como factor en el AFE.

En lo que respecta al tipo de programa usado, siguiendo la misma dinámica de la tradición versus nuevas recomendaciones (Lloret et al., 2014), el ACP fue realizado con SPSS, mientras que, el AFE fue realizado con el programa FACTOR (Lorenzo \& Ferrando, 2020). Este último programa es de acceso gratuito y es recomendado para análisis AFE en escalas policóricas (Freiberg, et al., 2013).

\section{Conclusiones}

El problema presentado en este artículo corresponde a la elección a la que se enfrentan los investigadores noveles al momento de seleccionar el método para la validación de una escala psicométrica. En especial, la decisión sobre si seguir la tradición o hacer uso de las nuevas recomendaciones.

Se planteó como objetivo entregar un ejemplo que muestra el contraste entre los resultados de las dos líneas de investigación. Así evidenciar las diferencias entre ambos análisis colaborando con la decisión a tomar.

Como conclusión primaria de este contraste, se demostró la diferencia entre los objetivos del ACP y AFE. EI primero, es útil cuando lo que buscamos es la reducción de variables, y, si se usa con una rotación que corresponda a las características de la muestra. En este caso, la escala al ser tipo Likert y por ser psicométrica se usó rotación Oblimin.

Por otra parte, el AFE, es el análisis que puede entregar información nueva sobre el comportamiento de la escala al definir factores. Este punto es muy importante para al investigador, al momento de tener como objetivo la búsqueda de variables latentes.

Como postura final, se busca que el investigador sea minucioso en el uso del lenguaje. Por lo tanto, ya no se habla de factores cuando se realiza un ACP, sino de componentes.

En segundo lugar, la elección del tipo de rotación determina cambios en el resultado. Ello se da tanto en el AFE como en el ACP. Por lo tanto, el investigador deberá considerar las recomendaciones de la literatura actual y debe analizar exhaustivamente las características de los datos para determinar el tipo de rotación. En esta investigación se comprueba tal punto, ya que, al usar rotación oblicua un componente desaparece por completo, mientras que no es así con la rotación ortogonal.

Podemos concluir que serán muchos los factores que influyen en la decisión del tipo de análisis y que el investigador tendrá la tarea de estar en permanente búsqueda de recomendaciones y/o análisis que ya se desaconsejan.

Otras aristas que se nombran en esta publicación, quedando como desafío a estudiar en el futuro, son los programas a usar dependiendo el tipo de análisis y el estudio del tipo de consistencia interna a usar para los diferentes análisis. 


\section{Agradecimiento}

Esta publicación contó con el apoyo de la Agencia Nacional de Investigación y Desarrollo / Programa Formación de Capital Humano Avanzado / Beca de Magíster en Chile para Profesionales de la Educación Folio 50180100

\section{Referencias bibliográficas}

Domínguez , S. (2012). Propuesta para el cálculo del Alfa Ordinal y Theta de Armor. Revista de Investigación En Psicología, 15(1), 213. https://doi.org/10.15381/rinvp.v15i1.3684

Ferrando P. \& Anguiano, C. (2010). El análisis factorial como técnica de investigación histórica. Papeles Del Psicólogo, 31(1), 13-33. Recuperado de: http://www.redalyc.org/articulo.oa?id=77812441003

Ferrando, P. J., Ledesma, R., \& Tosi, J. (2019). Uso del Análisis Factorial Exploratorio en RIDEP. Recomendaciones para Autores y Revisores The Use of Exploratory Factor Analysis in RIDEP . Guidelines for Authors and Reviewers Resumen. Revista Iberoamericana de Diagnóstico y Evaluación, 3(7600), 173180. Recuperado de: https://doi.org/https://doi.org/10.21865/RIDEP52.3.13

Freiberg, A. F., Stover, B. J., De la Iglesia, G., \& Fernández, M. (2013). Correlaciones policóricas y tetracóricas en estudios factoriales exploratorios y confirmatorios. Ciencias Psicológicas, VII, 151-164. Recuperado de: https://www.redalyc.org/pdf/4595/459545415005.pdf

Hayes, A. \& Coutts J. (2020) Use Omega Rather than Cronbach's Alpha for Estimating Reliability. But.... Communication Methods and Measures, 14:1, 1-24, Recuperado de: https://doi.org/10.1080/19312458.2020.1718629

Lloret, S., Ferreres, A., Hernández, A., \& Tomás, I. (2014). El análisis factorial exploratorio de los ítems: una guía práctica, revisada y actualizada. Anales de Psicología, 30(3), 1151-1169. Recuperado de: https://doi.org/10.6018/analesps.30.3.199361

Lloret, S., Ferreres, A., Hernández, A., \& Tomás, I. (2017). El análisis factorial exploratorio de los ítems: análisis guiado según los datos empíricos y el software. Anales de Psicología / Annals of Psychology, 33(2), 417432. https://doi.org/10.6018/analesps.33.2.270211

Lorenzo, U., \& Ferrando, P. J.(2017). Program FACTOR at 10: Origins, development and future directions. Psicothema 2017, Vol. 29, No. 2, 236-240. Recuperado de: doi: 10.7334/psicothema2016.304

Lorenzo-Seva, U., \& Ferrando, P. J. (2020). FACTOR (№ de versión 10.10.03). Windows. Tarragona (Spain): Rovira i Virgili University. Recuperado de: http://psico.fcep.urv.es/utilitats/factor

Panadero, E., \& Alonso-Tapia, J. (2014). ¿Cómo autorregulan nuestros alumnos? Modelo de Zimmerman sobre estrategias de aprendizaje. Anales de Psicología, 30(2), 450-462. Recuperado de: https://doi.org/10.6018/analesps.30.2.167221

Pérez, E., \& Medrano, L. (2014). Análisis factorial exploratorio : Bases conceptuales y metodológicas Análisis Factorial Exploratorio : Bases Conceptuales y Metodológicas Artículo de Revisión. Revista Argentina de Ciencias Del Comportamiento RACC, 2(July), 58-66. Recuperado de: https://dialnet.unirioja.es/servlet/articulo?codigo=3161108

Ventura-León, J., \& Caycho-Rodríguez, T. (2017). El coeficiente Omega: un método alternativo para la estimación de la confiabilidad. Revista Latinoamericana de Ciencias Sociales, Niñez y Juventud, 15(1),625- 
627.[fecha de Consulta 17 de Agosto de 2021]. ISSN: 1692-715X. Recuperado de: https://www.redalyc.org/articulo.oa?id=77349627039

Zimmerman, B. J. (2010). Becoming a Self-Regulated Learner: An Overview. Theory Into Practice, 5841(2002), 64-70. Recuperado de: https://doi.org/10.1207/s15430421tip4102_2

Zimmerman, B., Kitsantas, A., \& Campillo, M. (2005). Evaluación de la Autoeficacia Regulatoria: Una Perspectiva Social Cognitiva Barry J. Zimmerman*1, Anastasia Kitsantas**, Magda Campillo. Learning, 5, 1-21. Recuperado de: http://www.revistaevaluar.com.ar/51.pdf

\section{Anexos}

Anexo 1: Escala de autorregulación de los aprendizajes

\section{UNIVERSIDAD CATÓLICA DE LA SANTÍSIMA CONCEPCIÓN FACULTAD DE EDUCACIÓN PROGRAMA MAGÍSTER EN CIENCIAS DE LA EDUCACIÓN}

\section{VALIDACIÓN DE ESCALAS DE MEDICIÓN DE LOS PROCESOS DE AUTORREGULACIÓN DEL ESTUDIANTADO DE ENSEÑANZA MEDIA CHILENA DEL EJE DE LENGUAJE Y COMUNICACIÓN DE INVESTIGACIÓN}

\section{INSTRUCCIONES}

Las siguientes escalas miden la frecuencia con la que usted realiza procesos autorregulatorios en las actividades académicas de investigación de Lenguaje y Comunicación de la enseñanza media chilena.

$1^{\circ}$ Usted debe haber trabajado en clases uno de los siguientes objetivos de investigación. Léalo, de acuerdo a su curso y recuerde su investigación :

Primero y segundo medio

Objetivo de Aprendizaje 24: Realizar investigaciones sobre diversos temas para complementar sus lecturas o responder interrogantes relacionadas con el lenguaje y la literatura:

- delimitando el tema de investigación

- seleccionando páginas y fuentes según la profundidad y la cobertura de la información que buscan

- usando los organizadores y la estructura textual para encontrar información de manera eficiente

- evaluando si los textos entregan suficiente información para responder una determinada pregunta o cumplir un propósito

- evaluando la validez y confiabilidad de las fuentes consultadas

- jerarquizando la información encontrada en las fuentes investigadas

- registrando la información bibliográfica de las fuentes consultadas

- elaborando un texto oral o escrito bien estructurado que comunique sus hallazgos (Currículum nacional, 2019, p. 20)

Tercero medio y Cuarto Medio

Objetivo de Aprendizaje 8: Investigar sobre diversos temas para enriquecer sus lecturas y análisis, o para responder interrogantes propias de la asignatura:

- Seleccionando fuentes e información según criterios de validez y confiabilidad.

- Procesando la información mediante herramientas digitales o impresas.

- Comunicando sus hallazgos por medio de géneros (escritos, orales o audiovisuales) del ámbito educativo.

- Haciendo uso ético de la información investigada por medio de recursos de citación y referencia. (Currículum nacional, 2020, p. 42) 
$2^{\circ}$ Usted recibirá un vídeo que explica los procesos autorregulatorios de planificación, ejecución y reflexión de los aprendizajes y como usted puede aportar a esta investigación contestando el formulario que se le enviará online.

$3^{\circ}$ Este formulario primero requiere que des tu asentimiento de participación voluntario. Y el consentimiento informado de tu apoderado.

Estos implican que tu participación en esta investigación es voluntaria. Ello nos hace agradecer aún más tu colaboración al contribuir libremente con las Ciencias de la educación. Cabe señalar que la información recolectada es confidencial y es usada exclusivamente para fines académicos de esta investigación. No se verá afectada tu integridad personal ni académica.

$4^{\circ}$ Luego de ello, completa con tus datos y responde la escala de autorregulación. El formulario online contendrá la siguiente información:

Observe que hay tres partes las cuales corresponden a tres momentos de la investigación:

- Planificación, previa al desarrollo de la investigación

- Ejecución, que corresponde al desarrollo de la investigación

- Auto reflexión, que se realiza una vez terminada la investigación

\begin{tabular}{|l|}
\hline Nombre: \\
\hline Curso: \\
\hline Fecha: \\
\hline Edad: \\
\hline Colegio: \\
\hline Sexo: \\
\hline
\end{tabular}

Lea los ítems y marque la respuesta que le parece más adecuada a su experiencia en los trabajos de investigación.

Respuestas de la escala: Estas están ordenadas de MENOR frecuencia a MAYOR frecuencia.

\begin{tabular}{|c|c|c|c|c|c|c|}
\hline Nunca & Rara vez & Ocasionalmente & $\begin{array}{l}\text { Con mediana } \\
\text { frecuencia }\end{array}$ & Frecuentemente & $\begin{array}{c}\text { Muy } \\
\text { frecuentemente }\end{array}$ & Siempre \\
\hline $\begin{array}{c}\text { Nunca lo he } \\
\text { hecho. }\end{array}$ & $\begin{array}{c}\text { Casualmente o } \\
\text { fortuitamente lo } \\
\text { ha hecho en } \\
\text { muy pocas } \\
\text { ocasiones. }\end{array}$ & $\begin{array}{c}\text { Esporádica- } \\
\text { mente lo hace. }\end{array}$ & $\begin{array}{c}\text { Relativa } \\
\text { frecuencia o lo } \\
\text { ha hecho, pero } \\
\text { con menor } \\
\text { constancia }\end{array}$ & $\begin{array}{c}\text { Regularmente lo } \\
\text { hace }\end{array}$ & $\begin{array}{l}\text { Es un hábito casi } \\
\text { arraigado en el } \\
\text { comporta- } \\
\text { miento y es } \\
\text { constante }\end{array}$ & $\begin{array}{l}\text { Lo hace sin } \\
\text { excepción. }\end{array}$ \\
\hline
\end{tabular}


Escala de planificación

\begin{tabular}{|c|c|c|c|c|c|c|c|c|c|}
\hline & & $\begin{array}{l}\text { CONSIGNA: Antes de empezar la investigación en Lenguaje y } \\
\text { Comunicación... }\end{array}$ & $\stackrel{\overbrace = 5}{2}$ & 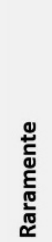 & 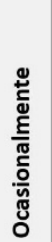 & 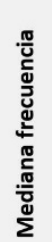 & 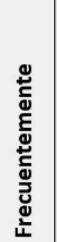 & 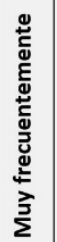 & 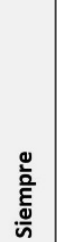 \\
\hline \multirow{3}{*}{$\begin{array}{l}\text { ANÁLISIS DE LA } \\
\quad \text { TAREA } \\
\text { establecimiento } \\
\text { de objetivos }\end{array}$} & 1 & 1.1.1.a. Me propuse un objetivo a lograr en esta investigación & & & & & & & \\
\hline & 2 & $\begin{array}{l}\text { 1.1.1.b. Analicé los criterios con los que evaluará el profesor. } \\
\text { (criterios de la pauta, rúbrica, entre otros) }\end{array}$ & & & & & & & \\
\hline & 3 & $\begin{array}{l}\text { 1.1.1.c. Me propuse lograr un nivel de perfección según los criterios de evaluación } \\
\text { del profesor. }\end{array}$ & & & & & & & \\
\hline \multirow{12}{*}{$\begin{array}{l}\text { ANÁLISIS DE LA } \\
\text { TAREA } \\
\text { planificación } \\
\text { estratégica }\end{array}$} & 4 & 1.1.2.a. Planifiqué un método de investigación para lograr el objetivo & & & & & & & \\
\hline & 5 & 1.1.2.b. Planifiqué la estrategia de investigación apropiada al objetivo & & & & & & & \\
\hline & 6 & 1.1.2.c. Planifiqué el paso a paso a seguir para realizar la investigación & & & & & & & \\
\hline & 7 & 1.1.2.d. Seleccioné el tema de investigación y lo delimité. & & & & & & & \\
\hline & 8 & $\begin{array}{l}\text { 1.1.2. e. Sabía cómo seleccionar fuentes de información de acuerdo a cobertura y } \\
\text { profundidad de ella. }\end{array}$ & & & & & & & \\
\hline & 9 & 1.1.2.f. Planifiqué la estructura del texto más coherente para la investigación & & & & & & & \\
\hline & 10 & 1.1.2.g. Planifiqué como evaluar la suficiencia de información de los textos. & & & & & & & \\
\hline & 11 & $\begin{array}{l}\text { 1.1.2.h. Sabía cómo evaluar la validez* de las fuentes consultadas. } \\
\text { *Validez. Significa que están relacionado al tema principal de la investigación. }\end{array}$ & & & & & & & \\
\hline & 12 & $\begin{array}{l}\text { 1.1.2.i. Sabía cómo evaluar la confiabilidad* de las fuentes consultadas. } \\
\text { *Confiabilidad. Significa si las fuentes son de fiar. }\end{array}$ & & & & & & & \\
\hline & 13 & 1.1.2.j. Planifiqué un método para organizar las fuentes investigadas. & & & & & & & \\
\hline & 14 & 1.1.2.k. Sabía cómo registrar la información bibliográfica de las fuentes consultadas. & & & & & & & \\
\hline & 15 & $\begin{array}{l}\text { 1.1.2.I. Planifiqué cómo comunicar mis hallazgos de la investigación (oral y/o } \\
\text { escrito). }\end{array}$ & & & & & & & \\
\hline \multirow{7}{*}{$\begin{array}{c}\text { CREENCIAS } \\
\text { AUTOMOTIVA- } \\
\text { DORAS }\end{array}$} & 16 & 1.2.1.a. Creía tener las capacidades necesarias para lograr el objetivo. & & & & & & & \\
\hline & 17 & 1.2.2. Tenía expectativas de tener éxito en la investigación. & & & & & & & \\
\hline & 18 & 1.2.3. a. Creía que era de mi interés dicho tema de investigación. & & & & & & & \\
\hline & 19 & 1.2. 3.b. Creía que era valioso el realizar dicha investigación. & & & & & & & \\
\hline & 20 & $\begin{array}{l}\text { 1.2.4.a. Creía que el realizar dicha investigación es (era) muy útil para lograr mi } \\
\text { meta que es incrementar mis aprendizajes. }\end{array}$ & & & & & & & \\
\hline & 21 & $\begin{array}{l}\text { 1.2.4.b. Creía que el resultado que obtenga servirá para verme competente frente } \\
\text { a los otros. }\end{array}$ & & & & & & & \\
\hline & 22 & $\begin{array}{l}\text { 1.2.4.c. Creía que un resultado satisfactorio evitaría malos juicios de los otros sobre } \\
\text { mi rendimiento. }\end{array}$ & & & & & & & \\
\hline
\end{tabular}


Escala de ejecución

\begin{tabular}{|c|c|c|c|c|c|c|c|c|c|}
\hline & & $\begin{array}{l}\text { CONSIGNA: Mientras realizo la investigación en Lenguaje } \\
\text { y Comunicación... }\end{array}$ & $\stackrel{\overparen{5}}{5}$ & 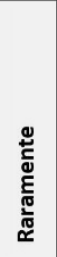 & 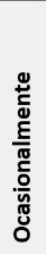 & 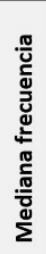 & 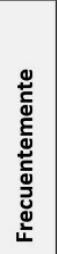 & 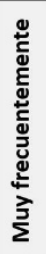 & 总 \\
\hline \multirow[t]{11}{*}{ AUTO OBSERVACIÓN } & 23 & $\begin{array}{l}\text { 2.1.1.a. Me pregunto si mi investigación está de acuerdo con lo que el } \\
\text { profesor me pidió. }\end{array}$ & & & & & & & \\
\hline & 24 & 2.1.1.c. Me preocupo de seguir las instrucciones del profesor. & & & & & & & \\
\hline & 25 & 2.1.1.d. Reviso si lo que he hecho trata el tema de investigación. & & & & & & & \\
\hline & 26 & $\begin{array}{l}\text { 2.1.1.e Reviso si las fuentes de información están de acuerdo a la } \\
\text { profundidad del tema. }\end{array}$ & & & & & & & \\
\hline & 27 & $\begin{array}{l}\text { 2.1.1.f. Reviso si el orden del texto que estoy usando es eficiente para } \\
\text { dicha investigación- }\end{array}$ & & & & & & & \\
\hline & 28 & $\begin{array}{l}\text { 2.1.1.g Evalúo si los textos entregan suficiente información para cumplir } \\
\text { el propósito de dicha investigación. }\end{array}$ & & & & & & & \\
\hline & 29 & $\begin{array}{l}\text { 2.1.1.h. Evalúo la validez de las fuentes consultadas. } \\
\text { *Validez. Significa que están relacionado al tema principal de la } \\
\text { investigación. }\end{array}$ & & & & & & & \\
\hline & 30 & 2.1.1.i. Evalúo la calidad de las fuentes consultadas. & & & & & & & \\
\hline & 31 & $\begin{array}{l}\text { 2.1.1.j. Voy revisando como estoy jerarquizando las fuentes para la } \\
\text { investigación. }\end{array}$ & & & & & & & \\
\hline & 32 & $\begin{array}{l}\text { 2.1.1.k. Monitoreo si registro la información bibliográfica de las fuentes } \\
\text { consultadas. }\end{array}$ & & & & & & & \\
\hline & 33 & $\begin{array}{l}\text { 2.1.2.i. Reviso si mi texto (oral o escrito) está organizado para comunicar } \\
\text { mis hallazgos. }\end{array}$ & & & & & & & \\
\hline \multirow[t]{7}{*}{$\begin{array}{l}\text { AUTOCONTROL } \\
\text { Metacognitivo }\end{array}$} & 34 & $\begin{array}{l}\text { 2.2.1.1. Me pregunto si lo que estoy haciendo me sirve para el objetivo } \\
\text { que el profesor me ha planteado. }\end{array}$ & & & & & & & \\
\hline & 35 & 2.2.1.2.a. Me doy auto instrucciones para mejorar y/o concentrarme. & & & & & & & \\
\hline & 36 & 2.2.1.2.b. Me doy órdenes para mejorar y/o concentrarme. & & & & & & & \\
\hline & 37 & $\begin{array}{l}\text { 2.2.1.3. Me organizo mentalmente con esquemas para visualizar la } \\
\text { información. }\end{array}$ & & & & & & & \\
\hline & 38 & $\begin{array}{l}\text { 2.2.1.4. Organizo los tiempos que necesito para la elaboración del texto } \\
\text { (oral u escrito) }\end{array}$ & & & & & & & \\
\hline & 39 & $\begin{array}{l}\text { 2.2.1.5. Puedo controlar los distractores del entorno escolar y de estudio } \\
\text { en el que realizo dicha investigación. }\end{array}$ & & & & & & & \\
\hline & 40 & 2.2.1.6. Si se presenta alguna duda pido ayuda al profesor o compañero. & & & & & & & \\
\hline \multirow{3}{*}{$\begin{array}{c}\text { AUTOCONTROL } \\
\text { motivacional }\end{array}$} & 41 & 2.2.2.1.a. Me incentivo para interesarme en la investigación & & & & & & & \\
\hline & 42 & $\begin{array}{l}\text { 2.2.2.1.b. Me auto motivo para mantenerme trabajando en la } \\
\text { investigación. }\end{array}$ & & & & & & & \\
\hline & 43 & $\begin{array}{l}\text { 2.1.2.1.2. Para auto motivarme: pienso en las consecuencias asociadas al } \\
\text { cumplimiento y no cumplimiento de la tarea }\end{array}$ & & & & & & & \\
\hline
\end{tabular}


Revista ESPACIOS. ISSN: 0798-1015

Escala de auto reflexión

\begin{tabular}{|c|c|c|c|c|c|c|c|c|c|}
\hline & & $\begin{array}{l}\text { CONSIGNA: Una vez terminada la investigación en Lenguaje } \\
\text { y Comunicación }\end{array}$ & $\stackrel{\overbrace{}}{5}$ & 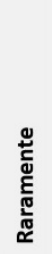 & 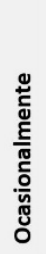 & 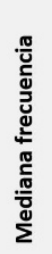 & 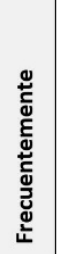 & 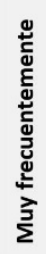 & 竞 \\
\hline \multirow[t]{7}{*}{ AUTO JUICIO } & 44 & 3.1.1. Me autoevalúo para ver si logré lo que el profesor me pidió. & & & & & & & \\
\hline & 45 & $\begin{array}{l}\text { 3.1.2. Pienso en las causas que ayudaron, o bien obstaculizaron su } \\
\text { realización. }\end{array}$ & & & & & & & \\
\hline & 46 & $\begin{array}{l}\text { 3.1.2.a. Reflexiono sobre cómo el esfuerzo que hice en la investigación } \\
\text { fueron causa de mi resultado final. }\end{array}$ & & & & & & & \\
\hline & 47 & $\begin{array}{l}\text { 3.1.2.b. Reflexiono sobre cómo mis capacidades para investigar fue causal } \\
\text { del resultado final. }\end{array}$ & & & & & & & \\
\hline & 48 & $\begin{array}{l}\text { 3.1.2.c. Reflexiono sobre cómo el grado de dificultad de la investigación fue } \\
\text { causal del resultado final. }\end{array}$ & & & & & & & \\
\hline & 49 & $\begin{array}{l}\text { 3.1.2.d. Reflexiono sobre cómo circunstancias externas no controlables* } \\
\text { fueron causal del resultado final. } \\
\text { *Suerte }\end{array}$ & & & & & & & \\
\hline & 50 & 3.1.3.e. Reflexiono sobre cómo el profesor influye en mi resultado final. & & & & & & & \\
\hline \multirow[t]{3}{*}{$\begin{array}{l}\text { AUTOREACCIÓN } \\
\text { cognitivo emocional }\end{array}$} & 51 & $\begin{array}{l}\text { 3.2.1. Autoevalúo si me siento satisfecho con el resultado de } \mathrm{mi} \\
\text { investigación. }\end{array}$ & & & & & & & \\
\hline & 52 & 3.2.2. Reflexiono en lo que debo mejorar para futuras investigaciones & & & & & & & \\
\hline & 53 & $\begin{array}{l}\text { 3.2.2. Reflexiono sobre cómo me siento con el resultado para decidir si } \\
\text { quiero volver a realizar una investigación. }\end{array}$ & & & & & & & \\
\hline
\end{tabular}




\section{Anexo 2: ACP Oblimin}

\section{Anexo 2 ACP oblimin}

Tabla

Ponderaciones de los componentes para el análisis de la escala de autorregulación de los aprendizajes

\begin{tabular}{|c|c|c|c|c|}
\hline & $\begin{array}{l}\text { Auto- } \\
\text { observación }\end{array}$ & Auto reflexión & $\begin{array}{l}\text { Análisis de } \\
\text { tarea }\end{array}$ & laAuto-control \\
\hline Rango & $1-7$ & $1-7$ & $1-7$ & $1-7$ \\
\hline Media & 50,9 & 42,3 & 33,7 & 35,3 \\
\hline Desviación estándar & 10,1 & 9,8 & 8,9 & 8,8 \\
\hline Sesgo & $-1,1$ &,- 86 &,- 60 &,- 84 \\
\hline Varianza explicada & 46.7 & 7,7 & 4,9 & 3,9 \\
\hline Alfa de cronbach & ,933 & 906 & ,898 & ,880 \\
\hline Omega & ,933 & ,907 & ,899 & ,880 \\
\hline
\end{tabular}

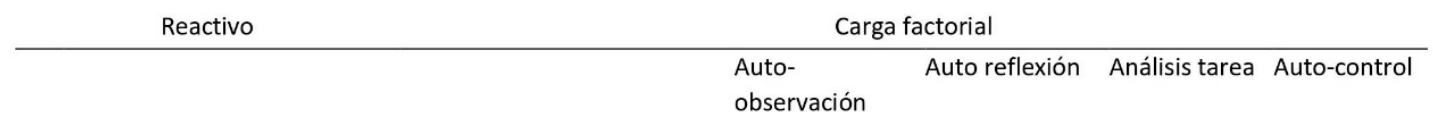

1 2.1.1.d. AUTO OBSERVACION- AUTOMONITOREO- Reviso si lo,787 que he hecho trata el tema de investigación.

2 2.1.1.h.AUTO OBSERVACION - AUTOMONITOREO-Evalúo la,728 validez de las fuentes consultadas. ${ }^{*}$ Validez. Significa que están relacionado al tema principal de la investigación.

3 2.1.1.a. AUTO OBSERVACION- AUTOMONITOREO- Me,711 pregunto si mi investigación está de acuerdo con lo que el profesor me pidió.

4 2.1.1.c. AUTO OBSERVACION- AUTOMONITOREO-Me,695 preocupo de seguir las instrucciones del profesor.

5 2.1.1.e. AUTO OBSERVACION-AUTOMONITOREO-Reviso si las,691 fuentes de información están de acuerdo a la profundidad del tema.

6 2.1.1.I. AUTO OBSERVACION- AUTOMONITOREO- Reviso si mi,684 texto (oral o escrito) esta organizado para comunicar mis hallazgos.

7 2.1.1.f. AUTO OBSERVACION- AUTOMONITOREO-Reviso si el,634 orden del texto que estoy usando es eficiente para dicha investigación.

8 2.1.1.g.AUTO OBSERVACION - AUTOMONITOREO-Evalúo si los,630 textos entregan suficiente información para cumplir el propósito de dicha investigación.

9 2.1.1.j. AUTO OBSERVACION - AUTOMONITOREO-Voy,431 revisando como estoy jerarquizando las fuentes para la investigación.

10 3.2.2. b AUTOREACCIÓN COGNITIVA Y EMOCIONAL--,216 Reflexiono sobre cómo me siento con el resultado para decidir si quiero volver a realizar una investigación.

11 3.1.2.a. AUTOJUICIO- CAUSAS- Reflexiono sobre como el,116 esfuerzo que hice fue causal de mi resultado final.

12 3.1.2.b. AUTOJUICIO- CAUSAS- Reflexiono sobre cómo mis,136 capacidades para investigar fue causal del resultado final. 
Continuación Tabla

Ponderaciones de los componentes para el análisis de la escala de autorregulación de los aprendizajes

Reactivo
13 3.1.2.c. AUTOJUICIO- CAUSAS- Reflexiono sobre cómo el
Carga factorial grado de dificultad de la investigación fue causal del resultado final.

14 3.1.2. AUTOJUICIO- CAUSAS- Pienso en las causas que ayudaron, o bien obstaculizaron su realización.

15 3.2.2.a. AUTOREACCIÓN COGNITIVA Y EMOCIONALReflexiono en lo que debo mejorar para futuras investigaciones

16 3.2.1. AUTOREACCIÓN COGNITIVA Y EMOCIONAL-,320 Autoevalúo si me siento satisfecho con el resultado de mi investigación.

17 3.1.1. AUTOJUICIO- OBJETIVOS- Me autoevalúo para ver si,302 logré lo que el profesor me pidió.

18 1.1.2.j. ANÁLISIS DE LA TAREA- PLANIFICACIÓN ESTRATÉGICA--,103 Planifiqué un método para organizar las fuentes investigadas.

19 1.1.2.a. ANÁLISIS DE LA TAREA- PLANIFICACIÓN ESTRATÉGICA- Planifiqué un método de investigación para lograr el objetivo

20 1.1.2.g. ANÁLISIS DE LA TAREA- PLANIFICACIÓN ESTRATÉGICA- Planifiqué como evaluar la suficiencia de información de los textos.

21 1.1.2.c. ANÁLISIS DE LA TAREA- PLANIFICACIÓN ESTRATÉGICA-,218 Planifiqué el paso a paso a seguir para realizar la investigación

22 1.1.2.e. ANÁLISIS DE LA TAREA- PLANIFICACIÓN,320 ESTRATÉGICA- Sabía cómo seleccionar fuentes de información de acuerdo a cobertura y profundidad de ella.

23 1.1.2.I. ANÁLISIS DE LA TAREA- PLANIFICACIÓN ESTRATÉGICA-,251 Planifiqué cómo comunicar mis hallazgos de la investigación (oral y/o escrito).

24 1.1.2.b. ANÁLISIS DE LA TAREA- PLANIFICACIÓN ESTRATÉGICA- Planifiqué la estrategia de investigación apropiada al objetivo

25 2.2.1.2.a. AUTOCONTROL - ESTR. METACOGNITIVA- Me doy,125 auto instrucciones para mejorar $y / o$ concentrarme.

26 2.2.1.2.b. AUTOCONTROL - ESTR. METACOGNITIVA- Me doy,130 órdenes para mejorar y/o concentrarme.

27 2.2.2.1.b. AUTOCONTROL - ESTR. MOTIVACIONALES-Me auto motivo para mantenerme trabajando en la investigación.

28 2.2.2.1.a. AUTOCONTROL - ESTR. MOTIVACIONALES- Me incentivo para interesarme en la investigación

29 2.2.1.3. AUTOCONTROL - ESTR. METACOGNITIVA- Me organizo mentalmente con esquemas para visualizar la información.

30 2.2.1.5. AUTOCONTROL - ESTR. METACOGNITIVA- Puedo controlar los distractores del entorno escolar y de estudio en el que realizo dicha investigación.

31 2.2.1.1. AUTOCONTROL - ESTR. METACOGNITIVA- Me,225 pregunto si lo que estoy haciendo me sirve para el objetivo que el profesor me ha planteado.

Método de extracción: ACP. Rotación oblicua, Oblimin.

,756

, 724

,714

,144

,612

$-, 148$

,602

,119

,784

,778

, 725

,585

,155

,583

$-, 126$

,553

,518

,278

,131

$-, 191$

,787

$-, 135$

, 744

,691

,141

,641

,162

,630

176

, 588

,180

,451 


\section{Anexo 3 ACP rotación varimax}

Tabla

Ponderaciones de los componentes para el análisis de la escala de autorregulación de los aprendizajes

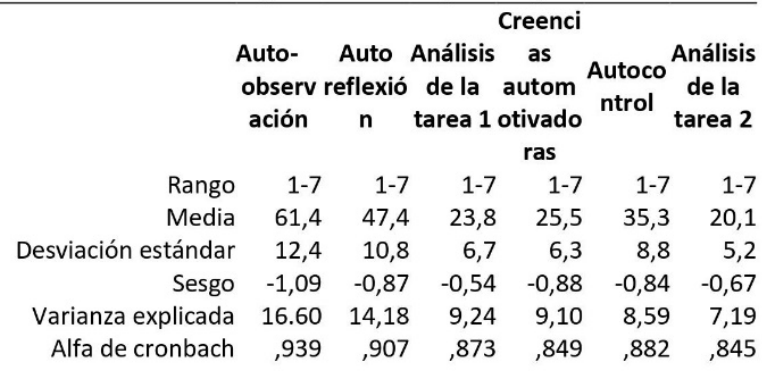

\begin{tabular}{|c|c|c|c|c|c|c|}
\hline & Reactivo & & Carg & factoria & & \\
\hline 1 & $\begin{array}{l}\text { 2.1.1.h.AUTO OBSERVACION - AUTOMONITOREO-Evalúo la validez } \\
\text { de las fuentes consultadas.*Validez. Significa que están,723 } \\
\text { relacionado al tema principal de la investigación. }\end{array}$ & 227, & 236, & 087 & 119, & 265, \\
\hline 2 & $\begin{array}{l}\text { 2.1.1.d. AUTO OBSERVACION- AUTOMONITOREO- Reviso si lo que }, 720 \\
\text { he hecho trata el tema de investigación. } \\
\text { 2.1.1.e. AUTO OBSERVACION- AUTOMONITOREO-Reviso si las }\end{array}$ & 211 & 166, & ,273 & 152, & 123 \\
\hline 3 & $\begin{array}{l}\text { fuentes de información están de acuerdo a la profundidad del,687 } \\
\text { tema. }\end{array}$ & 175 & 238 & ,219 & 241 & 123 \\
\hline 4 & $\begin{array}{l}\text { 2.1.1.a. AUTO OBSERVACION- AUTOMONITOREO- Me pregunto si } \\
\text { mi investigación está de acuerdo con lo que el profesor me pidió. }\end{array}$ & ,308 & , 163 & ,312 & 200 & 101 \\
\hline 5 & $\begin{array}{l}\text { 2.1.1.f. AUTO OBSERVACION-AUTOMONITOREO-Reviso si el orden } \\
\text { del texto que estoy usando es eficiente para dicha investigación. }\end{array}$ & 191, & 166 & ,114 & 367 & 178 \\
\hline 6 & $\begin{array}{l}\text { 2.1.1.I. AUTO OBSERVACION- AUTOMONITOREO- Reviso si mi } \\
\text { texto (oral o escrito) esta organizado para comunicar mis hallazgos. }{ }^{6},\end{array}$ & ,266 & ,089 & ,309 & 224, & 188 \\
\hline 7 & $\begin{array}{l}\text { 2.1.1.c. AUTO OBSERVACION-AUTOMONITOREO-Me preocupo de }, 634 \\
\text { seguir las instrucciones del profesor. }\end{array}$ & 186 & ,127 & ,278 & ,260 & 165 \\
\hline 8 & $\begin{array}{l}\text { 2.1.1.g.AUTO OBSERVACION - AUTOMONITOREO-Evalúo si los } \\
\text { textos entregan suficiente información para cumplir el propósito,621 } \\
\text { de dicha investigación. }\end{array}$ & 192, & 233 & ,255 & 185 & ,235 \\
\hline 9 & $\begin{array}{l}\text { 2.1.1.i. AUTO OBSERVACION - AUTOMONITOREO-Evalúo la calidad } \\
\text { de las fuentes consultadas. }\end{array}$ & ,223 & 156 & ,210 & 175 & ,403 \\
\hline 10 & $\begin{array}{l}\text { 2.1.1.k. AUTO OBSERVACIÓN - AUTOMONITOREO-Monitoreo si } \\
\text { registro la información bibliográfica de las fuentes consultadas. }{ }^{5}, 565\end{array}$ & 200 & ,349 & , 105 & 128 & ,342 \\
\hline 11 & $\begin{array}{l}\text { 2.1.1.j. AUTO OBSERVACION - AUTOMONITOREO-Voy revisando } \\
\text { como estoy jerarquizando las fuentes para la investigación. } \\
\text { 3.2.2. b AUTOREACCIÓN COGNITIVA Y EMOCIONAL- Reflexiono }\end{array}$ & 170, & ,526 & ,091 & 170, & 080, \\
\hline 12 & $\begin{array}{l}\text { sobre cómo me siento con el resultado para decidir si quiero volver,048 } \\
\text { a realizar una investigación. }\end{array}$ & ,761 & 167 & ,117 & 148 & ,075 \\
\hline 13 & $\begin{array}{l}\text { 3.1.2.a. AUTOJUICIO-CAUSAS- Reflexiono sobre como el esfuerzo } \\
\text { que hice fue causal de mi resultado final. }\end{array}$ & ,729 & ,148 & 175, & ,062 & 104 \\
\hline 14 & $\begin{array}{l}\text { 3.1.2.c. AUTOJUICIO- CAUSAS- Reflexiono sobre cómo el grado de } \\
\text { dificultad de la investigación fue causal del resultado final. }\end{array}$ & ,724 & 142 & 138 & 190 & ,064 \\
\hline 15 & $\begin{array}{l}\text { 3.1.2.b. AUTOJUICIO- CAUSAS- Reflexiono sobre cómo mis } \\
\text { capacidades para investigar fue causal del resultado final. }\end{array}$ & ,719 & ,117 & 113 & ,105 & 154 \\
\hline 16 & $\begin{array}{l}\text { 3.2.2.a. AUTOREACCIÓN COGNITIVA Y EMOCIONAL- Reflexiono en } \\
\text { lo que debo mejorar para futuras investigaciones }\end{array}$ & ,709 & 210 & ,053 & 277, & ,009 \\
\hline 17 & $\begin{array}{l}\text { 3.1.2. AUTOJUICIO- CAUSAS- Pienso en las causas que ayudaron, } 0 \\
\text { bien obstaculizaron su realización. }\end{array}$ & ,703 & ,039 & ,116 & 222 & 177 \\
\hline
\end{tabular}


Continuación Tabla

Ponderaciones de los componentes para el análisis de la escala de autorregulación de los aprendizajes $18 \begin{aligned} & \text { 3.2.1. AUTOREACCIÓN COGNITIVA Y EMOCIONAL- Autoevalúo si } \\ & \text { me siento satisfecho con el resultado de mi investigación. }\end{aligned}$ $19 \begin{aligned} & \text { 3.1.1. AUTOJUICIO- OBJETIVOS- Me autoevalúo para ver si logré lo } \\ & \text { que el profesor me pidió. }\end{aligned}$

3.1.3.e. AUTOJUICIO- CAUSAS- Reflexiono sobre cómo el profesor ${ }_{, 157}$ 1 influye en mi resultado final.

1.1.2.j. ANÁLISIS DE LA TAREA- PLANIFICACIÓN ESTRATÉGICA-

21 Planifiqué un método para organizar las fuentes investigadas. 1.1.2.g. ANÁLISIS DE LA TAREA- PLANIFICACIÓN ESTRATÉGICA-

22 Planifiqué como evaluar la suficiencia de información de los,255 textos.

1.1.2.c. ANÁLISIS DE LA TAREA- PLANIFICACIÓN ESTRATÉGICA-

23 Planifiqué el paso a paso a seguir para realizar la investigación 1.1.2.I. ANÁLISIS DE LA TAREA- PLANIFICACIÓN ESTRATÉGICA-

24 Planifiqué cómo comunicar mis hallazgos de la investigación (oral,365 y/o escrito).

25 1.1.2.a. ANÁLISIS DE LA TAREA- PLANIFICACIÓN ESTRATÉGICA-

Planifiqué un método de investigación para lograr el objetivo

26 1.2.2. CREENCIAS AUTOMOTIVADORAS- EXPECTATIVAS- Tenía, 327

expectativas de tener éxito en la investigación.

613 204

27 1.2.1. CREENCIAS AUTOMOTIVADORAS- EXPECTATIVAS- Creía 230

27 tener las capacidades necesarias para lograr el objetivo.

28 1.2.3. a. CREENCIAS AUTOMOTIVADORAS- INTERÉS- Creía que era ${ }_{, 213}$

8 de mi interés dicho tema de investigación.

29 1.2. 3.b. CREENCIAS AUTOMOTIVADORAS- VALOR- Creía que era ${ }_{, 229}$

valioso el realizar dicha investigación.

1.2.4.a. CREENCIAS AUTOMOTIVADORAS- PROPÓSITO- Creía que

30 el realizar dicha investigación es muy útil para lograr mi meta que,221 es incrementar mis aprendizajes.

2.2.1.2.a. AUTOCONTROL - ESTR. METACOGNITIVA- Me doy auto 1 instrucciones para mejorar $y / o$ concentrarme.

$32 \begin{aligned} & \text { 2.2.1.2.b. AUTOCONTROL - ESTR. METACOGNITIVA- Me doy } \\ & \text { órdenes para mejorar y/o concentrarme. }\end{aligned}$

2.2.2.1.b. AUTOCONTROL - ESTR. MOTIVACIONALES-Me auto 261

motivo para mantenerme trabajando en la investigación. 261

$4 \begin{aligned} & \text { 2.2.1.3. AUTOCONTROL - ESTR. METACOGNITIVA- Me organizo } \\ & \text { mentalmente con esquemas para visualizar la información. }\end{aligned}$

2.2.1.5. AUTOCONTROL - ESTR. METACOGNITIVA- Puedo controlar

35 los distractores del entorno escolar y de estudio en el que realizo,197 dicha investigación.

2.2.2.1.a. AUTOCONTROL - ESTR. MOTIVACIONALES- Me incentivo

36 para interesarme en la investigación 2.2.1.1. AUTOCONTROL - ESTR. METACOGNITIVA- Me pregunto si

37 lo que estoy haciendo me sirve para el objetivo que el profesor me,412 ha planteado.

1.1.2.k. ANÁLISIS DE LA TAREA- PLANIFICACIÓN ESTRATÉGICA-

38 Sabía cómo registrar la información bibliográfica de las fuentes,316 098 , 604 , 201 consultadas.

1.1.2.i. ANÁLISIS DE LA TAREA- PLANIFICACIÓN ESTRATÉGICA-

39 Sabía cómo evaluar la confiabilidad* de las fuentes,370 112 , 641 consultadas. ${ }^{*}$ Confiabilidad. Significa si las fuentes son de fiar.

\begin{tabular}{|c|c|c|c|c|}
\hline 604 & ,064 & ,087 & 206 & ,087 \\
\hline 563, & 309, & ,235 & 051 & -112 \\
\hline 201, & 744 & 122 & 163, & 198, \\
\hline 193, & 673, & 275 & ,064 & 244 \\
\hline 125, & 599, & ,319 & 105, & 278, \\
\hline 130, & ,561 & 249 & 209, & 208, \\
\hline 224 & 419, & 290 & 195, & 398, \\
\hline 246 & 174, & 666 & 166, & 198, \\
\hline 144 & 112, & 635 & 225 & 359, \\
\hline 254 & 239, & 615 & ,068 & 116, \\
\hline 241 & ,435 & ,529 & 234 &,- 036 \\
\hline 272 & 353, & ,505 & 154, & 096, \\
\hline 309, & 013 & 123 & 682, & 061 \\
\hline 353, & ,027 & 208 & 659, & 121, \\
\hline 285, & 154, & ,374 & ,596 & 055, \\
\hline 183, & 323, & ,070 & ,592 & 157, \\
\hline 200 & 212 & -,039 & 570, & 439, \\
\hline 319 & 313, & ,440 & ,532 &,- 098 \\
\hline 354 & 175, & 227 & 429, & ,034 \\
\hline 098 & 307 & -,023 & 201 & 642, \\
\hline 112, & 134, & ,372 & 068, & 641, \\
\hline
\end{tabular}

1.1.2.e. ANÁLISIS DE LA TAREA- PLANIFICACIÓN ESTRATÉGICA-

40 Sabía cómo seleccionar fuentes de información de acuerdo a,255 113 348 , 530 , 051 , cobertura y profundidad de ella.

1.1.2.h. ANÁLISIS DE LA TAREA- PLANIFICACIÓN ESTRATÉGICA-

41 Sabía cómo evaluar la validez* de las fuentes consultadas.*Validez.
Significa que están relacionado al tema principal de $\quad$ 127 a investigación.

Método de extracción: ACP. rotación ortogonal, Normalized Varimax.

Esta obra está bajo una Licencia Creative Commons Attribución-NoCommercial 4.0 International

\section{(c) $\mathrm{BY}-\mathrm{NC}$}

